\title{
Trial sequential analysis suggested the potential overestimated effect of carbonic anhydrase inhibitor for respiratory failure and metabolic alkalosis
}

\author{
Meng-Si Luo ${ }^{1}$, Hui-Zi Li ${ }^{2}$, Guan-Jiang Huang ${ }^{3^{*}}$ (D) and Lun $\mathrm{Wu}^{1 *}$ \\ This comment refers to the article available at https://doi.org/10.1186/s13054-018-2207-6
}

\begin{abstract}
Meta-analyses of randomized controlled trials (RCTs) used to be considered as the optimum evidence to guide clinical practices. Generally, a high-quality meta-analysis with conclusive information should meet the minimum requirements of a well-conducted $\mathrm{RCT}$, which includes prospective protocol development, limitation of bias, and adequate sample size [1]. Conversely, meta-analyses based on limited RCTs may trigger the potential overestimation of the authentic intervention effect owing to weak statistical power [1]. More interestingly, increasing studies indicated that pooled results with false positive were frequently existed in published meta-analyses including many Cochrane ones [2, 3].

Trial sequential analysis (TSA) was introduced to monitor potential random error, false positive, and false negative in meta-analyses of RCTs [3]. Moreover, it was recommended that TSA should be
\end{abstract}

performed to assess the "imprecision" of outcomes of interest in the Grading of Recommendations Assessment, Development and Evaluation (GRADE) [4]. A recent meta-analysis indicated that carbonic anhydrase inhibitor (CAI) may have a positive effect on respiratory failure and metabolic alkalosis [5]. Considering that limited trials with small information size included in the study, we assumed that the effect of CAI for respiratory failure and metabolic alkalosis may be overestimated. Subsequently, we performed TSA for one of outcomes (i.e. $\mathrm{PaCO}_{2}$ ) with the most included RCTs to estimate whether the evidence is enough reliable and credible. TSA in Fig. 1 showed that the cumulative $Z$-curve did not cross the trial sequential monitoring boundary for benefit and the required information size boundary, which suggested that the current evidence (the positive effect of CAI on $\mathrm{PaCO}_{2}$ ) was inconclusive.

\footnotetext{
*Correspondence: hgj719471594@zju.edu.cn; lunwuzhongshan@outlook.com ${ }^{3}$ Department of Otorhinolaryngology, The Second Affiliated Hospital, School of Medicine, Zhejiang University, 88 Jiefang Road, Hangzhou 310009, Zhejiang, China

'Department of Anesthesiology, Zhongshan Hospital of Traditional Chinese Medicine, Affiliated to Guangzhou University of Chinese Medicine, 3 Kangxin Road, Zhongshan 528400, Guangdong, China

Full list of author information is available at the end of the article
} 


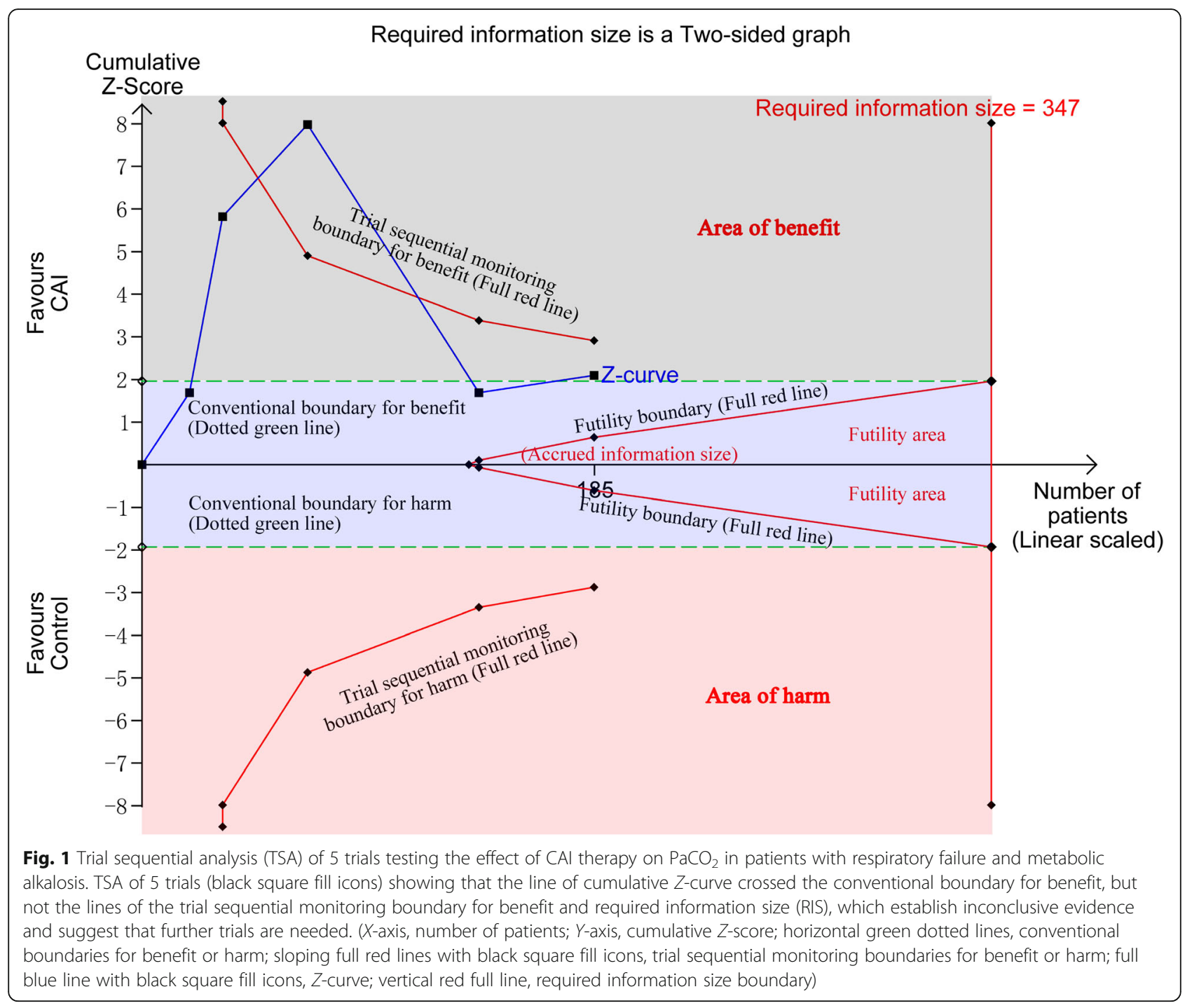

In addition, TSA on $\mathrm{PaCO}_{2}$ showed that the required information size (347 patients) is not reached due to weak statistical power. So, the effects of CAI therapy for patients with respiratory failure and metabolic alkalosis may very likely be overrated.
Collectively, for meta-analyses of RCTs with limited information size, TSA is a good choice to monitor the potential overestimation of the overall pooled effect. Furthermore, it is worthwhile to further discussion whether TSA should be routinely performed in meta-analyses of RCTs.

\section{Authors' response}

Bassem Y. Tanios, Pierre K. Bou-Khalil, Samir S. Mallat and Elie A. Akl

We thank Meng-Si Luo et al. for their interest in our work, and for highlighting one the limitations of the literature in this field, namely the limited number of published randomized controlled trials (RCTS). In our conclusion, we do acknowledge this important limitation and the need for larger, well designed, and RCTs addressing clinically important outcomes such as mortality, duration of hospital stay, and duration of mechanical ventilation. [5]

Meng-Si Luo et al. propose the use of Trial Sequential Analysis (TSA) to assess the 'imprecision' of outcomes. In a recent expert panel consensus statement, the Cochrane scientific committee recommended against the use of sequential methods for the main analysis, or to 
draw main conclusions. The statement encourages authors to interpret evidence based on the estimated magnitude of the effect of intervention and its uncertainty (usually quantified using a confidence interval), rather than focusing primarily on the rejection of the null hypothesis of no treatment effect (like that used by Meng-Si Luo et al. in their TSA) [6].

Our approach was consistent with the recent Cochrane recommendations, as we did not draw binary interpretation of the effect estimate as "significant" or "non-significant". Instead, we presented our results using a confidence interval and assessed heterogeneity using the I2 statistic, then used the GRADE methodology to rate the certainty of evidence. In applying GRADE, we judged imprecision as a reason for rating down the certainty of evidence for all the clinically important outcomes. [6, 7] If we were to analyze the certainty of evidence for the change in $\mathrm{PaCO} 2$ using the GRADE methodology, we would have similarly rated down the certainly of evidence for imprecision (and further down due to significant heterogeneity resulting in a low certainty evidence). Indeed, our meta-analysis found a mean reduction of - $4.98 \mathrm{mmHg}$ in the carbonic anhydrase inhibitors group ( $95 \%$ CI -9.66 to -0.3 ; I2 = 95\%). The confidence interval includes both values indicating a clinically significant benefit and values indicating no effect [8].

One of the main reasons Cochrane adopted the above recommendation is to "support the decision maker and end user by providing the best and latest evidence, but that interpretation of that evidence should be left to the user to make within their own context." We are happy our approach was consistent with that recommendation given the aim of our review is to support clinicians providing care to critically ill patients.

\section{Abbreviations}

CAl: Carbonic anhydrase inhibitor; GRADE: Grading of Recommendations Assessment, Development and Evaluation; RCT: Randomized clinical trial; TSA: Trial sequential analysis

\section{Acknowledgements}

Not application.

\section{Funding}

Not applicable.

Availability of data and materials

Not applicable.

\section{Authors' contributions}

M-SL was responsible for the conception of the letter and wrote the manuscript. H-ZL and G-JH conceived and wrote this manuscript. LW was responsible for the conception of the letter and revised the manuscript. All authors had read and approved this final manuscript.
Consent for publication

Not applicable.

\section{Competing interests}

The authors declare that they have no competing interests.

\section{Publisher's Note}

Springer Nature remains neutral with regard to jurisdictional claims in published maps and institutional affiliations.

\section{Author details}

'Department of Anesthesiology, Zhongshan Hospital of Traditional Chinese Medicine, Affiliated to Guangzhou University of Chinese Medicine, 3 Kangxin Road, Zhongshan 528400, Guangdong, China. ${ }^{2}$ Department of Orthopaedics, The Fifth Affiliated Hospital of Sun Yat-Sen University, 52 Meihua East Road, Xiangzhou District, Zhuhai 519000, Guangdong, China. ${ }^{3}$ Department of Otorhinolaryngology, The Second Affiliated Hospital, School of Medicine, Zhejiang University, 88 Jiefang Road, Hangzhou 310009, Zhejiang, China.

Received: 20 December 2018 Accepted: 6 March 2019

Published online: 24 April 2019

\section{References}

1. Pogue J, Yusuf S. Overcoming the limitations of current meta-analysis of randomised controlled trials. Lancet. 1998;351:47-52.

2. Brok J, Thorlund $\mathrm{K}$, Gluud $\mathrm{C}$, et al. Trial sequential analysis reveals insufficient information size and potentially false positive results in many meta-analyses. J Clin Epidemiol. 2008;61:763-9.

3. Imberger G, Thorlund K, Gluud C, et al. False-positive findings in Cochrane meta-analyses with and without application of trial sequential analysis: an empirical review. BMJ Open. 2016;6:e011890.

4. Jakobsen JC, Wetterslev J, Winkel P, et al. Thresholds for statistical and clinical significance in systematic reviews with meta-analytic methods. BMC Med Res Methodol. 2014;14:120.

5. Tanios BY, Omran MO, Noujeim C, et al. Carbonic anhydrase inhibitors in patients with respiratory failure and metabolic alkalosis: a systematic review and meta-analysis of randomized controlled trials. Crit Care. 2018;22:275.

6. Agendas and Minutes: Cochrane Scientific Committee available from Cochrane.org https://methods.cochrane.org/sites/default/files/public/ uploads/tsa_expert_panel_guidance_and_recommendation_final.pdf.

7. Guyatt G, Oxman AD, Akl EA, Kunz R, Vist G, Brozek J, et al. GRADE guidelines: 1. Introduction-GRADE evidence profiles and summary of findings tables. J Clin Epidemiol. 2011;64(4):383-94.

8. Guyatt GH, Oxman AD, Kunz R, Brozek J, Alonso-Coello P, Rind D, et al. GRADE guidelines 6. Rating the quality of evidence--imprecision. J Clin Epidemiol. 2011;64(12):1283-93. 